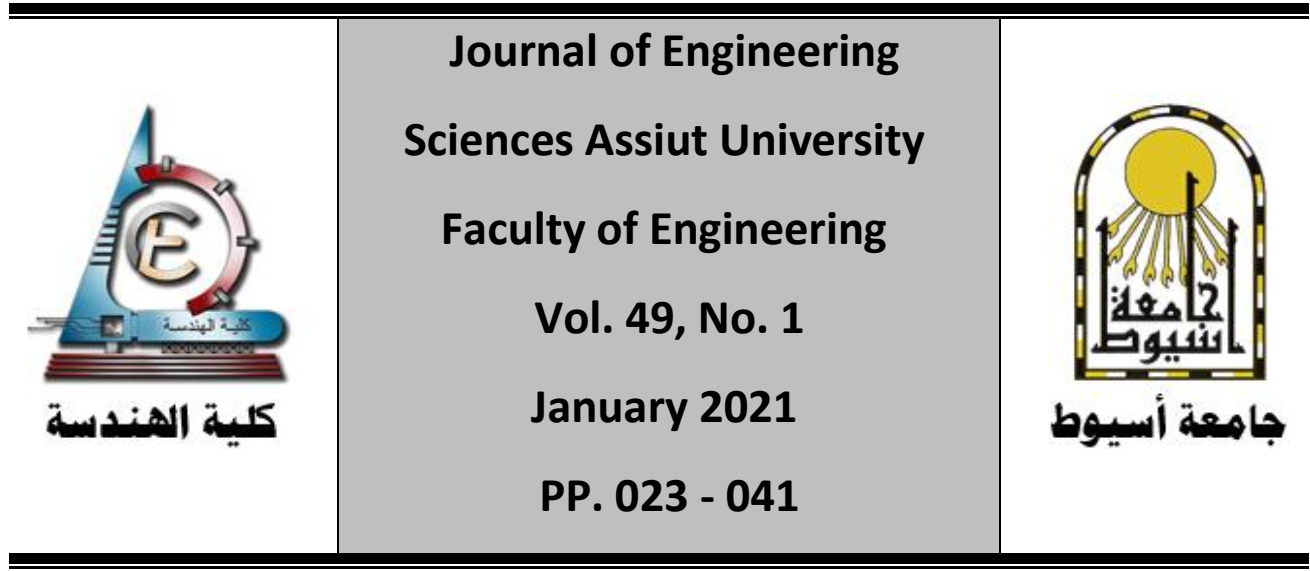

\title{
ANALYSIS OF CHANNEL CODING PERFORMANCE FOR IMAGE TRANSMISSION OVER COOPERATIVE RELAY NETWORKS
}

\section{Safwat Mohamed Ramzy ${ }^{1}$, Fatma Khairy Mohamed ${ }^{2}$, Usama Sayed Mohamed ${ }^{3}$}

\footnotetext{
${ }^{1}$ Assistant Professor of Department of Electrical Engineering, Sohag University, Sohag Egypt Email: safwat.ramzy@eng.sohag.edu.eg

${ }^{2}$ Department of Electrical Engineering, Sohag University, Sohag Egypt

Email: fatemakhairy207@yahoo.com

${ }^{3}$ Professor of Department of Electrical Engineering, Assiut University, Assiut Egypt Email: usama@aun.edu.eg
}

Received 8 October 2020; Revised 29 November 2020; Accepted 8 December 2020

\begin{abstract}
:
In this paper, we examine and investigate the effects of noise-induced transmission errors on the performance of image transmission over cooperative relay networks with various error-correction capabilities. First, the relationships between lengths of the code, peak signal-to-noise ratio, and signal-to-noise ratio are analyzed. Second, the comparison between the performances of different channel coding is introduced in conjunction with the set partitioning in hierarchical trees algorithm as a source code. Genetic algorithm, as an efficient optimization technique, is applied for solving the problem of optimal unequal error protection method to reduce the transmission rate and to improve the transmitted image quality. Many useful and significant results that are introduced in this paper can be used in the enhancement of image transmission over the cooperative networks. Compared to traditional
\end{abstract}


methods, the suggested method is more practical for the transmission of images with high-quality via smart devices.

Keywords: Image transmission; $\mathrm{BCH}$ coding; Cooperative Relay network; MAP.

\section{Introduction}

With the evolution of multimedia communications and the rapid outgrowth of the Internet, images have become the most common data exchanged [1]. The transmission links normally are subject to different sources of fading effects and noise with the limited bandwidth of the transmission channels [2]. Therefore, the necessity of image protection, image compression, and fault tolerance is a critical issue in reducing the required transmission bandwidth and transmission time. Several techniques have been suggested to minimize the bit rate and induced errors with achieving higher received image quality [3]. However, it is difficult to decode and reconstruct the image under fault conditions while maintaining acceptable quality. Error Correction Codes (ECC) [4-6] help in detecting and correcting errors in the transmitted data. ECC diagrams have a limit on the maximum number of errors that can be addressed or corrected. The Set partitioning in hierarchical trees (SPIHT) algorithm [7] is an image coding algorithm that is modified in [8] to transmit images through various channels with the strong transmission. The error protection system leans towards the important data in the transmitted image and video which is the case in most unequal protection techniques (UEP) [8-9]. An extensive review of the large variation of error protection schemes is introduced in [10-11]. For an efficient error detecting and correcting code, the Bose-Chaudhuri-Hocquenghem $(\mathrm{BCH})$ code was used in [12] because of its strong ability to randomize and detonate correction. An adaptive $\mathrm{BCH}$ scheme was proposed in [12] to choose the correction capabilities that offer the reconstructed image at high quality at a given SNR.

Recently, cooperative relay [13] is rapidly gaining interest in this multiintermediary device (relays) that can cooperate to improve the overall relays network performance. They benefit from the nature of transmission provided by the wireless medium, whereby the transmitted signals can be received and processed by any device in the coverage area of the transmitter [13-15]. The main idea of network coding [16] is that 
information that comes from multiple sources is mixed (encoded), to boost the reliability of decoding at the destination. The literature has suggested many communication protocols [17], such as decoding and forwarding (DF), amplification and forwarding (AF), and compression and forward $(\mathrm{CF})$. In this work, we consider the DF protocol as a communication protocol. The decoding scheme proposed in [16] presents the maximum a posterior decoder (MAP) for cooperative communication networks as the relay devices combine data received from sources to create the parity bits that are forwarded to the destination.

The Genetic algorithm (GA), as an advanced optimization technique, is derived from the notion of natural evolution based on the Darwinian theory of "survival of the fittest" by Holland [18]. In this paper, GA is utilized to get the optimum rate that minimizes the image distortion function. To conquer the specific disadvantages of static channel protection; there is a need for adaptive protection. Adaptive protection [20] adds redundancy data according to the channel behaviors and the importance of the different parts of the data. The authors in [21] introduced an adaptive scheme for image transmission over a cooperative network. They achieved both energy efficiency and image quality under the complex wireless channel. The real-time transmission of images through a multimode fiber (MMF) is still a challenging research work. One method completes image transmission by measuring and controlling the full complex field of the MMF [22]. To improve the image data transmission efficiency in the satellite communication systems, a class of riteless codes which can provide unequal error protection (UEP) property and equal water-fall region performance is proposed in this paper [23]. This paper analyzes the effects of noise on image transmission through the cooperative relay network when the channel coding is used. First, the relationships between bit error rate, signal-to-noise ratio (SNR), and code lengths are analyzed. Moreover, the transmission performance of $\mathrm{BCH}$ codes associated with different schemes is analyzed. This paper is arranged as follows. The problem formulation is introduced in Section 2. The proposed scheme is presented in Section 3. Numerical results and discussions are mentioned in Section 4. Conclusions are mentioned in Section 5. 


\section{Problem Formulation}

In this section, the problem of FEC using channel coding will be presented. Assume the image is encoded by the modified SPIHT coder [8]. The generated bit stream is divided into a series of packets. Let $\Delta D_{-} i>0$ indicates the expected reduction in distortion if the $\mathrm{i}$-th packet is decoded correctly. The overall distortion can be written as follows [7]:

$$
\mathrm{D}(\mathrm{L})=\mathrm{D}_{0}-\sum_{\mathrm{i}=1}^{\mathrm{L}} \prod_{v=1}^{i} \varphi_{v}\left(\mathrm{r}_{v}\right) \Delta D_{i}
$$

where $\mathrm{D} \_0$ is the expected distortion when the rate is zero, $\varphi_{v}\left(\mathrm{r}_{v}\right)$ is the probability that the $\mathrm{v}$-th source packet is received correctly when it is sent by a rate of $r_{v}$. $L$ is the number of the sent packets. Let $P_{v}$ be the undetected error probability of the v-th packet. Then $\varphi_{v}\left(\mathrm{r}_{v}\right)$ can be written as follows:

$$
\varphi_{v}\left(\mathrm{r}_{v}\right)=1-\mathrm{P}_{v}
$$

Then equation (1) can be rewritten as follows:

$$
\mathrm{D}(\mathrm{L})=\mathrm{D}_{0}-\sum_{\mathrm{i}=1}^{\mathrm{L}} \prod_{v=1}^{\mathrm{i}}\left(1-\mathrm{P}_{v}\right) \Delta \mathrm{D}_{\mathrm{i}}
$$

The undetected error probability of the $\mathrm{v}$-th packet bit $\mathrm{P}_{\mathrm{v}}$ relies on the rate assigned to $\mathrm{v}$-th packet $\mathrm{r}_{v}$ as shown in the next section. Therefore, the problem is to reduce the expected distortion $\mathrm{D}(\mathrm{L})$ according to the overall transmission rate limitations. We can formulate the problem as follows:

$$
\operatorname{Min} \underbrace{\mathrm{D}(\mathrm{L})}_{\mathrm{r}} \text { subject to } \sum_{v=1}^{L} r_{v} \leq \mathrm{R}
$$

where $\mathrm{R}$ is the total transmission rate.

\subsection{Cooperative Relay Network}

Without loss of generality, the cooperative network [16] shown in Fig. 1 is considered in this paper, that is consisting of two relays, two sources, and a destination. A transmission channel is assigned to each source. In the proposed work, the DF protocol is considered as the cooperation protocol. Firstly, the relays decode the data sent by the sources and then the relays combine them to create the parity bits. Data are sent using 
binary phase-shift keying (BPSK) modulation scheme. All channels are assumed to be Rayleigh fading channels with additive Gaussian noise (AWGN). The received signals at the destination at four consecutive channels are given by [16]

$$
y_{\mathrm{i}}=\mathrm{g}_{\mathrm{i}} \mathrm{s}_{\mathrm{i}} \sqrt{\mathrm{d}_{\mathrm{i}}^{-\mathrm{z}} \mathrm{E}_{\mathrm{i}}}+\mathrm{w}_{i} \quad \mathrm{i}=1,2,3,4
$$

where

- $y_{\mathrm{i}}$ is the received signal from the $\mathrm{i}-$ th source for $\mathrm{i}=1,2$ and from the $(i-2)-$ th relay for $\mathrm{i}=3,4$.

- $g_{i}$ is the channel fading gain between the $i-$ th source and the destination for $\mathrm{i}=1,2$ and that between $(i-2)-$ th relay and the destination for $\mathrm{i}=3,4$.

- $\mathrm{s}_{\mathrm{i}} \in\{+1,-1\}, \mathrm{i}=1,2$, is the BPSK modulation of the $\mathrm{i}$ - th source bits, $\mathrm{s}_{\mathrm{i}} \in\{+1,-1\}, \mathrm{i}=3,4$ is the BPSK modulation of the ( $\left.\mathrm{i}-2\right)-$ th relay for $\mathrm{i}=3,4$.

- $d_{i}$ is the distance between the $i-$ th source and the destination for $\mathrm{i}=$ 1,2 and that between $(\mathrm{i}-2)$ - th relay and the destination for $\mathrm{i}=$ 3,4 .

- $\mathrm{E}_{\mathrm{i}}$ is the transmit energy of the $\mathrm{i}-$ th source for $\mathrm{i}=1,2$ and $(\mathrm{i}-2)-$ th relay for $\mathrm{i}=3,4$.

- $\mathrm{z}$ is the path loss exponent.

- $\mathrm{w}_{i}$ is the white Gaussian noise with zero mean and variance $\mathrm{N}_{0} / 2$ between the $\mathrm{i}-$ th source and the destination for $\mathrm{i}=1,2$ and that between $(\mathrm{i}-2)$ - th relay and the destination for $\mathrm{i}=3,4$.

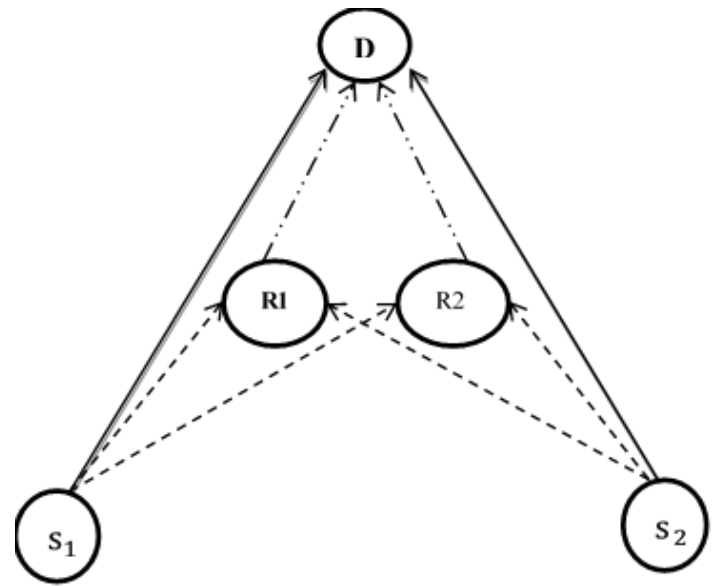

Fig. 1 Cooperative network model [16] 
The received signal can be written in a matrix form as follows [18]

$$
\mathrm{Y}=\mathrm{GS}+\mathrm{W}
$$

where the received vector $\mathrm{Y}=\left[\mathrm{y}_{1} \mathrm{y}_{2} \mathrm{y}_{3} \mathrm{y}_{4}\right]^{\mathrm{T}}$, the transmitted vector $S=\left[\begin{array}{llll}s_{1} & s_{2} & s_{3} & s_{4}\end{array}\right]^{T}$, the noise vector $W=\left[\begin{array}{llll}w_{1} & w_{2} & w_{3} & w_{4}\end{array}\right]^{T}$, and $\mathrm{G}$ is given by

$$
\mathrm{G}=\left[\begin{array}{cccc}
\mathrm{g}_{1} \sqrt{\mathrm{d}_{1}^{-\mathrm{z}} \mathrm{E}_{1}} & 0 & 0 & 0 \\
0 & \mathrm{~g}_{2} \sqrt{\mathrm{d}_{2}^{-\mathrm{z}} \mathrm{E}_{2}} & 0 & 0 \\
0 & 0 & \mathrm{~g}_{3} \sqrt{\mathrm{d}_{3}^{-\mathrm{z}} \mathrm{E}_{3}} & 0 \\
0 & 0 & 0 & \mathrm{~g}_{4} \sqrt{\mathrm{d}_{4}^{-\mathrm{z}} \mathrm{E}_{4}}
\end{array}\right]
$$

The MAP decoder [16] is used at the destination as it estimates the transmitted code word according to

$$
\begin{aligned}
\hat{\mathrm{c}} & =\arg \max _{\mathrm{w}_{\mathrm{i}}} \frac{\mathrm{P}\left(\mathrm{c}_{\mathrm{i}}\right) \frac{1}{\left(\pi \mathrm{N}_{0}\right)^{\frac{3}{2}}} \mathrm{e}^{-\left\|\mathrm{Y}-\mathrm{GS}_{\mathrm{i}}\right\|^{2}}}{\mathrm{~N}_{0}} \\
& =\arg \min _{\mathrm{c}_{\mathrm{i}}}\left\|\mathrm{Y}-\mathrm{GS}_{\mathrm{i}}\right\|^{2}-\mathrm{N}_{0} \log \left(\mathrm{P}\left(\mathrm{c}_{\mathrm{i}}\right)\right)
\end{aligned}
$$

where $\mathrm{P}\left(\mathrm{c}_{\mathrm{i}}\right)$ is the transmitted probability of the codeword $\mathrm{c}_{\mathrm{i}}$ that depends on the channel conditions between the sources and the relays. $\mathrm{P}_{\mathrm{u}}$ is the upper bound for the probability of error that can be found in [16] and it is used later for optimizing the channel coding process.

\subsection{Forward Error Correction with BCH Codes}

In this paper, the $\mathrm{BCH}$ code [4] is used to correct errors escaped from the MAP decoder inside the cooperative network. $\mathrm{BCH}$ is an errorcorrecting code where a block code is defined by $(n, k)$, where $n$ represents the size of the codeword and $k$ represents the size of source bits, therefore, $(n-k)$ is the number of parity-check bits. A parameter $t$ represents the maximum errors in the $\mathrm{BCH}$ codeword that can be corrected by the $\mathrm{BCH}$ code.

$$
\mathrm{n}-\mathrm{k} \leq \mathrm{mt}
$$

where $\mathrm{n}=2^{\mathrm{m}}-1, \mathrm{~m}$ is an integer positive number. 
It is important to note that the larger the value $(\mathrm{n}-\mathrm{k})$, the more errorcorrecting capability and the less size of the transmitted stream. However, the effectiveness of any error technique depends mainly on the channel conditions. Each source packet that has $\mathrm{k}$ bits is protected by $(\mathrm{n}-\mathrm{k})$ parity bits producing a packet of size $\mathrm{n}$ using $\mathrm{BCH}$ code. Let the data are transmitted through the cooperative network described above with a bit error rate $\mathrm{P}_{\mathrm{u}}$. Hence the undetected error probability of the $v$ - th packet bits, $\mathrm{P}_{v}$ using $\mathrm{BCH}$ code can be estimated as follows [20]:

$$
\mathrm{P}_{v}=2^{-\mathrm{mt}_{v}} \sum_{\mathrm{s}=0}^{\mathrm{t}_{v}}\left(\begin{array}{l}
\mathrm{n} \\
\mathrm{s}
\end{array}\right) \sum_{\mathrm{h}=\mathrm{t}_{v}+1}^{\mathrm{n}}\left(\begin{array}{l}
\mathrm{n} \\
\mathrm{h}
\end{array}\right) \mathrm{P}_{\mathrm{u}}^{\mathrm{h}}\left(1-\mathrm{P}_{\mathrm{u}}\right)^{\mathrm{n}-\mathrm{h}}
$$

where $\mathrm{t}_{v}$ represents the maximum the number of errors that can be corrected by the $\mathrm{BCH}$ code in the $v-$ th block or packet. Substituting from (10) into (3) yields:

$$
\mathrm{D}(\mathrm{L})=\mathrm{D}_{0}-\sum_{\mathrm{i}=1}^{\mathrm{L}} \prod_{v=1}^{\mathrm{i}}\left(1-2^{-\mathrm{mt}_{v}} \sum_{\mathrm{s}=0}^{\mathrm{t}_{v}}\left(\begin{array}{l}
\mathrm{n} \\
\mathrm{s}
\end{array}\right) \sum_{\mathrm{h}=\mathrm{t}_{v}+1}^{\mathrm{n}}\left(\begin{array}{l}
\mathrm{n} \\
\mathrm{h}
\end{array}\right) \mathrm{P}_{\mathrm{u}}^{\mathrm{h}}\left(1-\mathrm{P}_{\mathrm{u}}\right)^{\mathrm{n}-\mathrm{h}}\right) \Delta \mathrm{D}_{\mathrm{i}}
$$

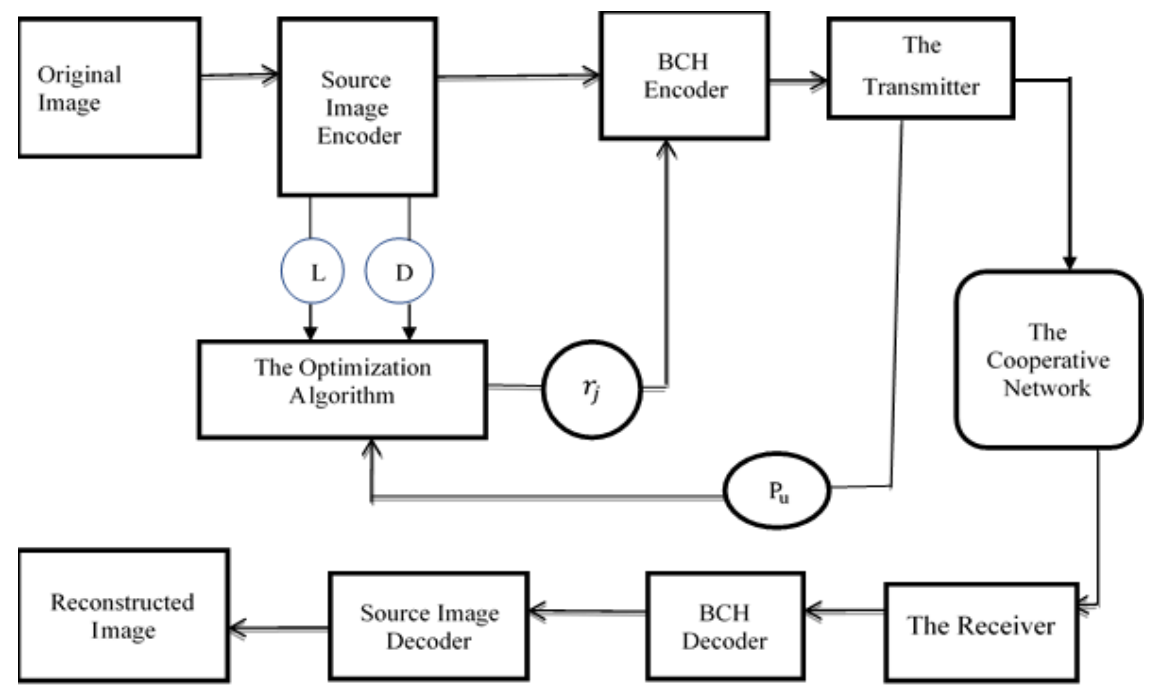

Fig. 2 The Proposed Scheme for UEP 
Hence, the expected distortion expression in (11) for the total transmission rate constraint can be minimized. The total redundancy bits can be estimated as follows:

$$
R_{d}=\sum_{i}^{L} m t_{i}=R-\text { (image data length from source coder) }
$$

Equation (12) represents the constraint that the optimization algorithm considers. Therefore, after the optimization process, the $\mathrm{BCH}$ coder can determine the needed redundancy $(n-k)$ to code every packet of the image data.

\subsection{Genetic Algorithm (GA)}

GA was inspired by the concept of natural evolution based on the Darwinian "survival of the fittest" theory by Holland [18]. The starting point for GA is the selection of the candidate solutions represented by the chromosomes, which in GA are like the population. A new population or a new generation was created by the old population. The choose of solutions or new offspring is suitable for their fitness. Hence, better solutions have greater production opportunities. The process is repeated until conditions are determined [19]. The advantages of GA over other common optimization techniques can be summarized [25] as follows:

a. GA can avoid trapping into local optima because Candidate solutions are better than normal techniques, as filter solutions have many points over normal techniques that have only one point.

b. GA is characterized by values and is not dependent on differentiation of other auxiliary elements.

c. GA is based on parameter set coding. Thus, the search process is applied to solve both integer and discrete problems.

d. Selection and crossover are used by GA to randomly select parents, so that exploration is performed to produce the best new offspring.

e. GA is used here to find the optimum rate $r_{j}$ of $j-t h$ image data packet that achieves the minimum overall distortion $\mathrm{D}(\mathrm{L})$.

\section{The Proposed Scheme}

The block diagram of the proposed scheme is shown in Fig. 2. In the beginning, the image is encoded by the modified SPIHT coder [8]. The output from the modified SPIHT coder consists of four groups. Each group is different in its importance from each other. The importance of 
each packet in each group is analyzed in [8] then $\Delta D_{i}$ is estimated for each packet in the bit steam. The proposed scheme can be summarized in the following points:

1) SPIHT image coding is modified to generate four groups of bit streams linked in order of importance, and then the output data is split into series of packets.

2) The reduction in distortion $\Delta D_{i}$ is approximately estimated.

3) Based on the number of the source packet, $\Delta \mathrm{D}_{\mathrm{i}}$ of each packet and the bit error probability of the cooperative network $\mathrm{P}_{\mathrm{u}}$, the optimization algorithm, that is GA, is used to generate the optimum value of $r_{j}$ of each packet subject to the total transmission rate constraint.

4) Based on the values of $\mathrm{r}_{j}$ for $j=1, \ldots \ldots L$ determined by the optimization algorithm, the $\mathrm{BCH}$ encoder encodes each packet according to their significance.

5) The coded data is sent to the receiver through the relay network.

6) The MAP decoder at the receiver estimates the transmitted data.

7) The $\mathrm{BCH}$ decoder decodes the transmitted data, and it tries to correct the errors due to the transmission channel.

8) Finally, the image is reconstructed by the SPIHT decoder.

\section{Numerical Results and Discussions}

The proposed scheme is tested on a cooperative network with fading channels and AWGN channel. The mean of the square of fading gains are assumed to be unity. The tested image is a LENA image $512 \times 512$ grey scale. First, the original images are decomposed into sub-images using the wavelet transform with a 7-tap high-pass filter and a 9-tap lowpass filter [24]. In the proposed test, we only use 6 layers in the wavelet domain. Then the wavelet coefficients are converted to the source bit stream with a bit rate of $0.4 \mathrm{bpp}$ using the modified SPIHT. The source bit stream is divided into four groups then each group is divided into packets. Initially, we tried to place the results of the unequal error protection (UEP) method in a comparison form with of the results of the equal error protection (EEP) method. Diversity is done through a network of one relay or two relays with different SNR and BER as will be shown in the simulation results. Fig. 3 shows an example of the channel rate allocation of UEP scheme produced by GA. More redundancy bits are assigned to the most significant data as shown in Fig. 
3. Fig. 4 shows the average PSNR of the decoded LENA image against the transmission rate for equal error protection (EEP) and unequal error protection (UEP) coding method that transmitted through a one-relay cooperative network. The bit error rate for cooperative relay network $\mathrm{P}_{\mathrm{u}}$ is 0.0636 and the received SNR is $5 \mathrm{~dB}$. In the case of the EEP, all packets are protected by the same size of redundancy bits. In the case of UEP, each packet is protected by different redundancy bits according to its importance that is determined by the optimization algorithm. The total transmission rate is the same in the cases EEP and UEP. In the case of UEP, the GA optimization is used to determine the required channel coding rate $r_{j}$ for each packet which helps the $\mathrm{BCH}$ encoder to determine the parameters needed to work $(n, k)$. The results obtained by the UEP scheme outperform those obtained by the EEP scheme by $6 \mathrm{~dB}$ that reflects the high performance of the proposed scheme.

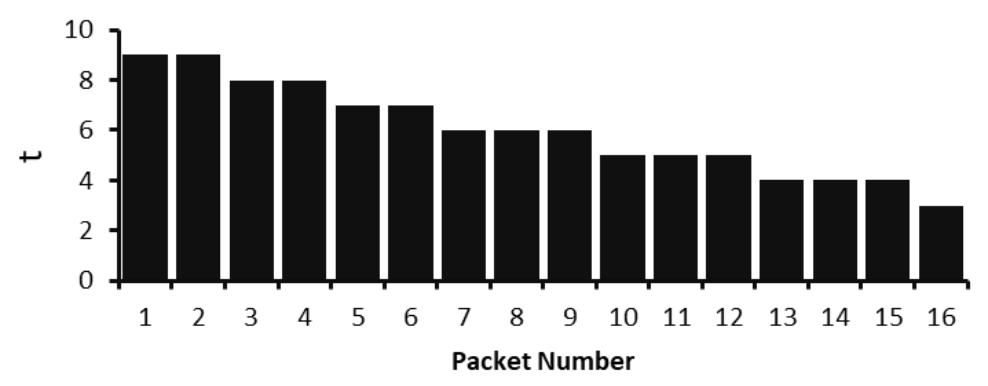

Fig. 3 Channel rates of UEP scheme

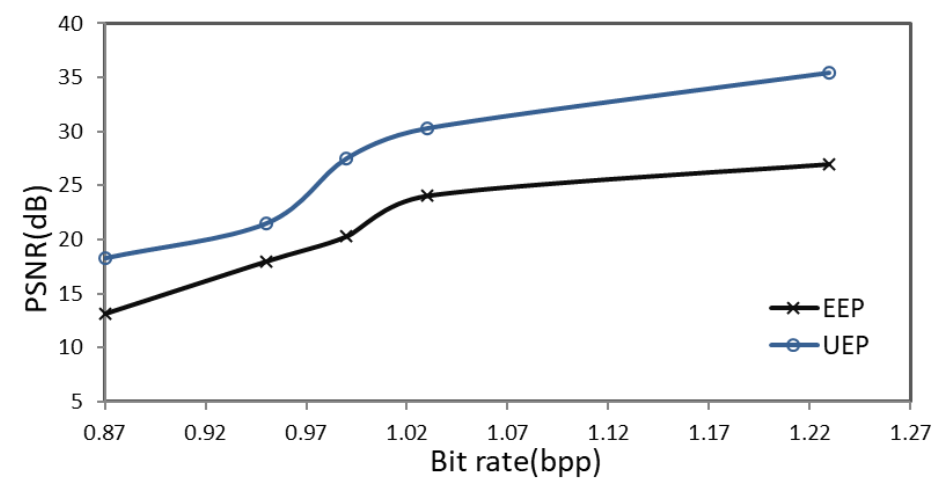

Fig. 4 The PSNR of a one-relay network at 5dB SNR for the BCH code.

In the case of using two relays, Fig. 5 illustrates the average PSNR of the decoded LENA image as a function of the transmission rate for EEP and 
UEP coding method. The bit error rate for cooperative relay network $\mathrm{P}_{\mathrm{u}}$ is 0.03 and the received SNR is $5 \mathrm{~dB}$. Because the data is transmitted over two relays cooperative network that is reflected on the values of BER, the resulting PSNR of the decoding image is better than the obtained PSNR in one-relay cooperative network. Fig.6 shows the required transmission rate in three cases against the received SNR. The three cases are without any diversity, with aiding of one relay, and with aiding of two relays. With EEP channel coding $(\mathrm{BCH})$, it is desired to achieve PSNR of $35 \mathrm{~dB}$ in the three cases at different values of the received SNR. It is clear that the more using relays, the less required transmission rate.

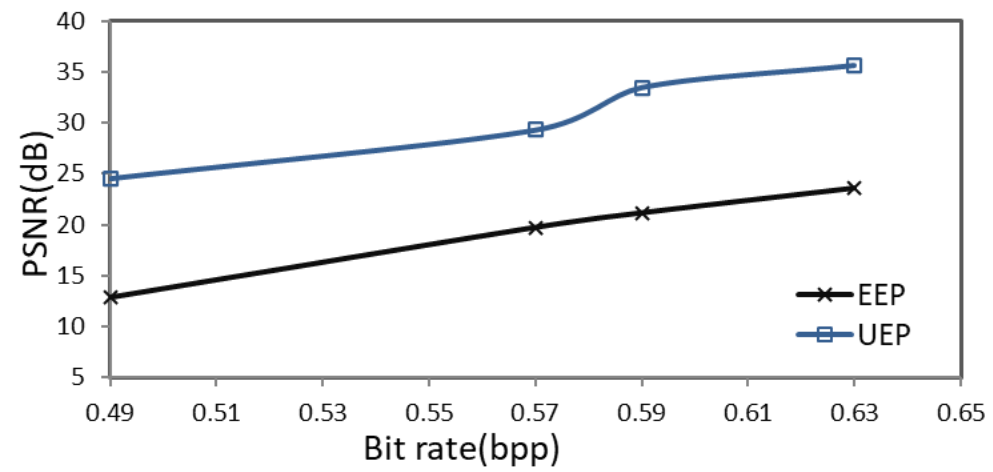

Fig. 5 The PSNR of two-relay network at 5dB SNR for BCH code.

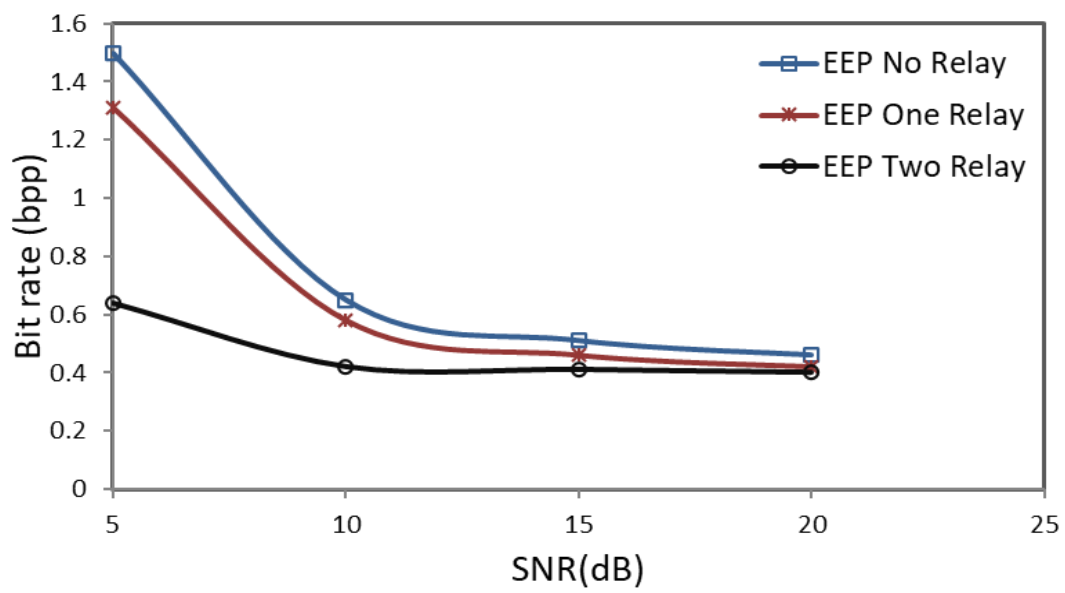

Fig. 6 The transmission rate against the received SNR of different networks.

Another interesting issue that has been investigated in this study is the effect of the length of the block of $\mathrm{BCH}$ code (n). Is there a value of 
$\mathrm{BCH}$ code block length having the best performance than others? Indeed, the answer to this question is yes. Fig. 7 shows the average PSNR performance for the two-relays network with a different block length $\mathrm{n}$ for $\mathrm{BCH}$ code at $\mathrm{SNR}=5 \mathrm{db}$. Fig. 8 shows that at the same transmission rate and low BER, the resulting PSNR differs for the three cases of a different block length. It is found that the resulting PSNR is higher at a block length of 1023 than that PSNR at block length of 2047 and PSNR at a block length of 16383 . The interpretation of this issue is very easy. If the number of transmitted block errors exceeded $\mathrm{t}$ errors, the $\mathrm{BCH}$ decoder will not be able to correct the error in the transmitted block despite the block length. The errors occur randomly during transmission. Due to the fading channel nature, the errors are not uniformly distributed between data packets. So, the error may be lager in a block than others so the BER $\mathrm{P}_{\mathrm{u}}$ may be very different than $\left(\frac{t}{n}\right)$. Because the error in a small block length is significant than the error in a large block length, we may get a high PSNR with using a small block length. For example, if four packets are sent with block length $\mathrm{n}=1023$, assuming packet $1,2,3,4$ has an error of $5,15,10,25$ bits during the transmission process respectively, and $(t)=15$, packet $1,2,3$ will be received correctly and packet 4 will be received with error. Now, if we decide to send the same data using two packets only of length $\mathrm{n}=2047$, and if the packets 1,2 has an error of 20,35 bits respectively, and $(t)=30$ then packet " 1 " will be received correctly and packet " 2 " is received with error. Notice packet "2" in the second case includes the data of packet 3, 4 of the first case which means that data of packet 3 is received correctly when $n=1023$ and it is received with error when $n=2047$. This explains why the block length is very important in the $\mathrm{BCH}$ coding process. Fig. 8 confirms the same idea as shown in Fig. 7.

However, in Fig. 8, the best value of (n) is 8191 because SNR $=10 \mathrm{~dB}$ so the network has better BER performance than in Fig. 6. It is shown that the best value of block length $(\mathrm{n})$ relies on the BER value of the cooperative network $\left(P_{u}\right)$. i.e., the larger value of BER $\left(P_{u}\right)$, the smaller value of the best $\mathrm{BCH}$ block length (n) . 


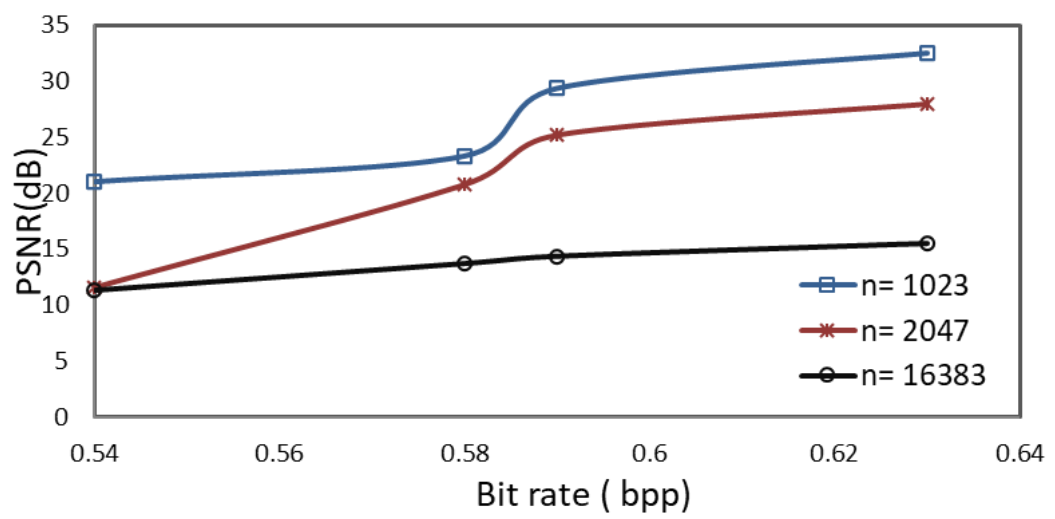

Fig. 7 The PSNR of a two-relay network at different codeword lengths of $\mathrm{BCH}$ code at $5 \mathrm{~dB}$ SNR.

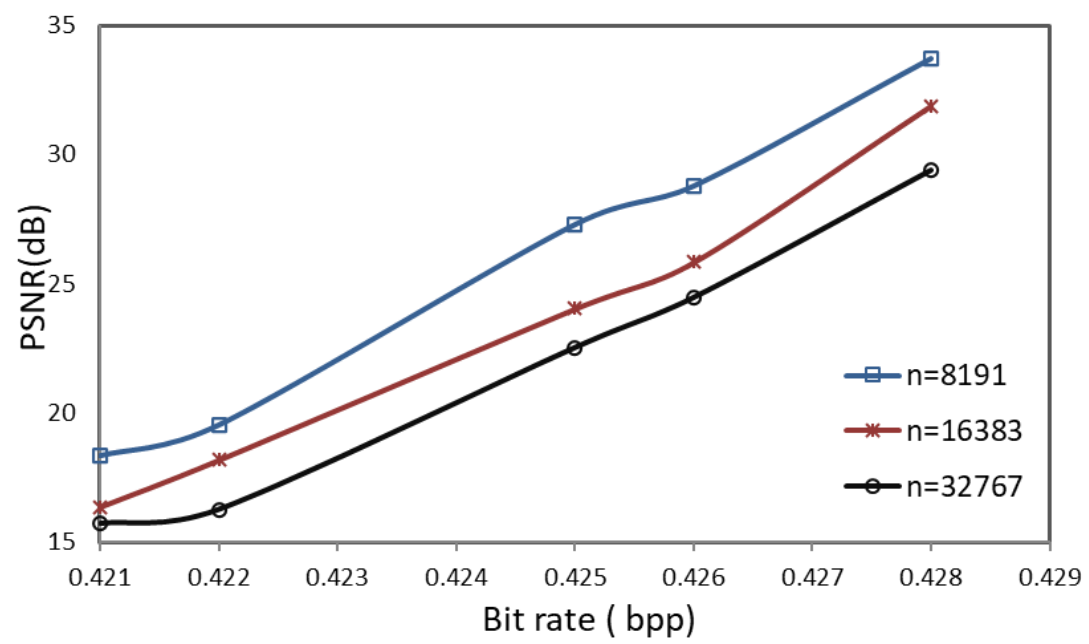

Fig. 8 the PSNR of two-relay network for different codeword lengths of $\mathrm{BCH}$ code at $10 \mathrm{~dB}$ SNR.

Another interesting issue that has been investigated in this study is the comparison between the $\mathrm{BCH}$ code and the rate-compatible punctured convolutional code (RCPC) code [4]. Fig. 9 shows the transmission rate in bpp performance using a one-relay network with $\mathrm{BCH}$ and RCPC channel coding at $\mathrm{SNR}=5,10,15,20$ and the output PSNR is $35 \mathrm{~dB}$ for different values of SNR. It is shown that, in the case of a high BER and a low SNR, the RCPC code performance is better than the performance of the $\mathrm{BCH}$ code because it requires less transmission rate than the $\mathrm{BCH}$ code to achieve PSNR $=35 \mathrm{db}$. With decreasing BER value and increasing SNR values, BCH code performance is better than RCPC performance 
because it requires a low transmission rate compared to RCPC code. Fig. 10 shows the transmission rate in bpp performance using a two-relay network with $\mathrm{BCH}$ and $\mathrm{RCPC}$ channel coding at $\mathrm{SNR}=5,10,15,20$ at output PSNR of $35 \mathrm{~dB}$ for different values of SNR.

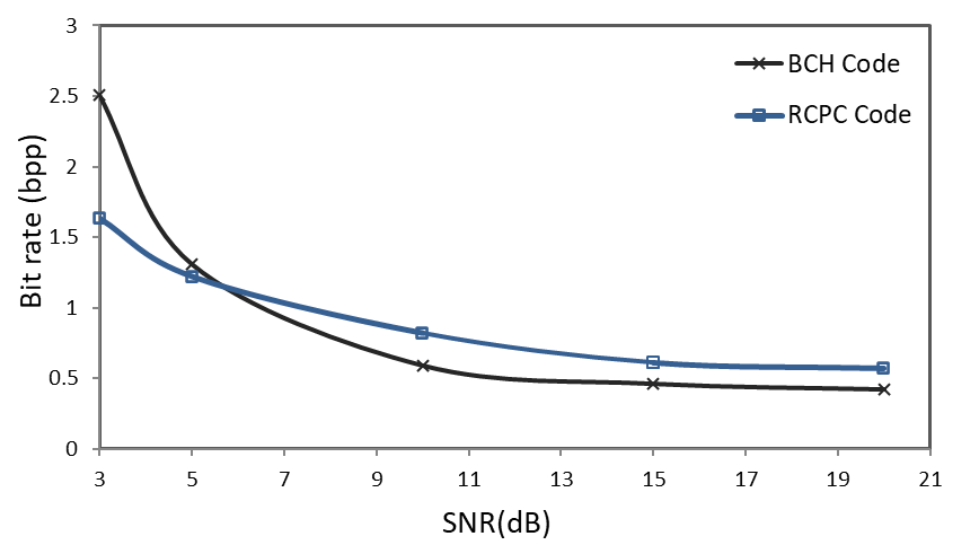

Fig. 9 Comparison between $\mathrm{BCH}$ and $\mathrm{RCPC}$ performance of one-relay network.

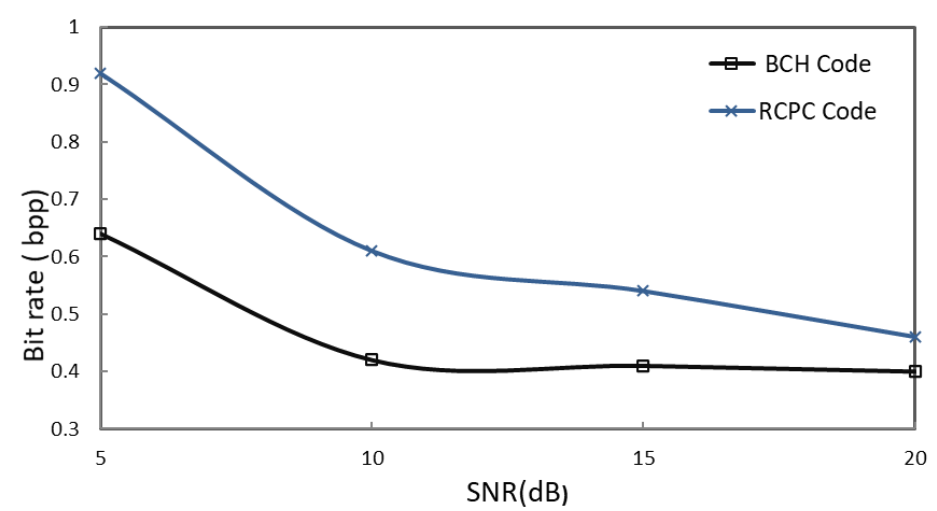

Fig. 10 Comparison between $\mathrm{BCH}$ and RCPC performance of two-relay network.

The $\mathrm{BCH}$ code performance outperforms the RCPC performance with improving in the BER performance. Fig.11 and Fig.12 show the average PSNR performance for two-relay and three relays network respectively, under $\mathrm{BCH}$ and $\mathrm{RCPC}$ channel coding with the transmission rate in bpp. It is shown that PSNR in case of using $\mathrm{BCH}$ is larger than in in case of using RCPC. 


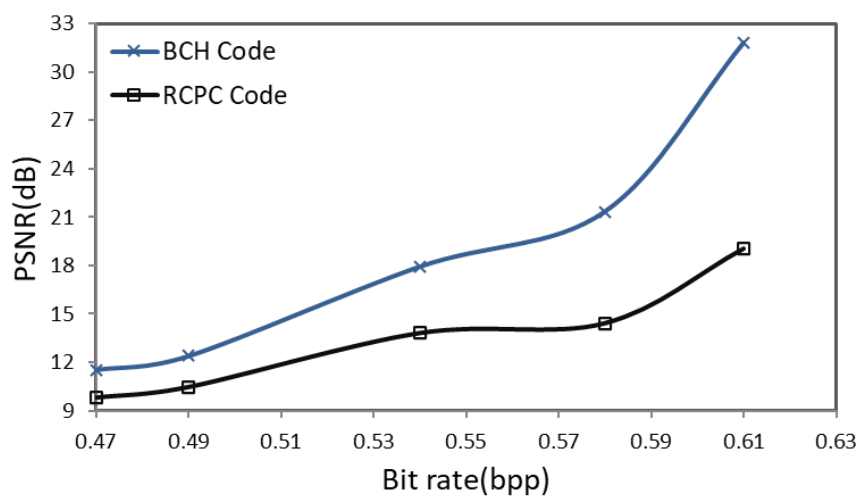

Fig. 11 The PSNR of a two-relay network for BCH and RCPC at 5dB SNR.

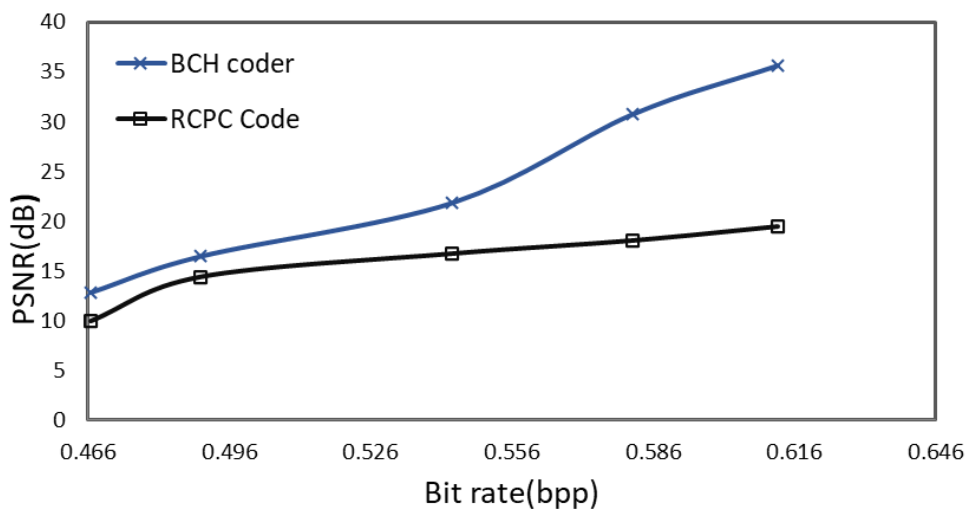

Fig. 12 The PSNR of a three-relay network for BCH and RCPC at 5dB SNR.

Another interesting issue that has been investigated in this study is the comparison between the $\mathrm{BCH}$ code and the low-density parity check matrix (LDPC) code [25]. Fig. 13 shows the transmission rate in bpp performance using a one-relay network with BCH and LDPC channel coding at $\mathrm{SNR}=3,5,7,10,15,20$ at PSNR of 35dB for different values of SNR. It is shown that, in the case of a high BER and a low SNR, the LDPC code performance is better than the performance of the $\mathrm{BCH}$ code because it requires less transmission rate than the $\mathrm{BCH}$ code to achieve $\mathrm{PSNR}=35 \mathrm{db}$. With decreasing BER value and increasing SNR values, $\mathrm{BCH}$ code performance is better than LDPC performance because it requires a low transmission rate compared to the LDPC code. 


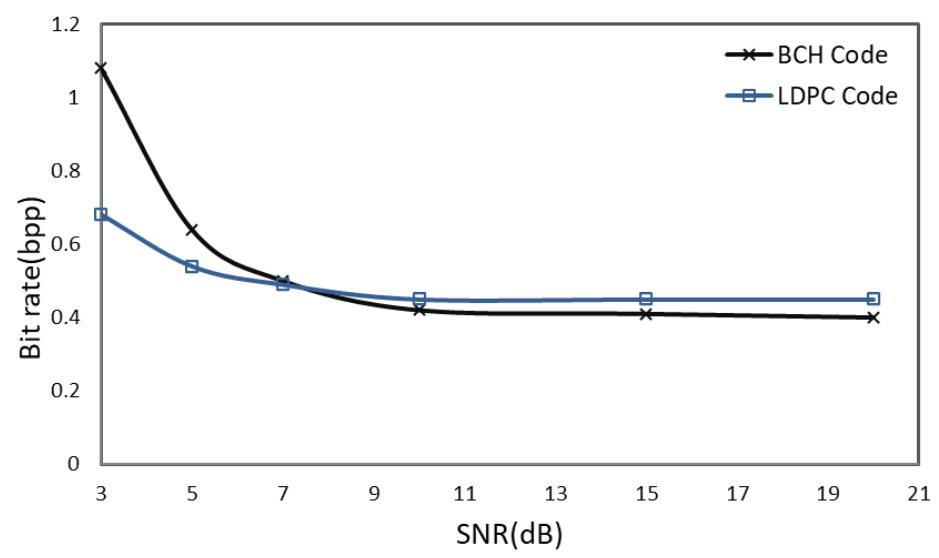

Fig. 13 Comparison between $\mathrm{BCH}$ and LDPC performance of two-relay network.

\section{Conclusions}

In this work, the study and analysis of three channel coding for image transmission over cooperative networks are introduced. The system used for the analysis is proposed for the transmission of image streams over the fading channel through a cooperative relay network. Genetic algorithm is considered as an optimization technique is applied for finding the optimum channel allocation rates of the coding in all packets. The inputs to the optimization algorithm are the number of source packets, the bit error rate (BER), and the model of the distortion function. The $\mathrm{BCH}$ code is used to protect the image by adding redundancy bits according to the number of errors that can be corrected according to the used optimization technique. The optimum packet size is used with different BER with two relays. RCPC code performance is investigated in the case of using two relays, one relay, and without using any relays, and its performance is compared with $\mathrm{BCH}$ code performance with image PSNR and transmission rate. The simulation results show that $\mathrm{BCH}$ code performance is better than RCPC and LDPC performance in the case of a high received signal to noise ratio. However, the RCPC and LDPC performance is better than the $\mathrm{BCH}$ performance at high BER. The research work is done using MATLAB, other Special Network Simulation Tools/SW Packages will be considered for future work. 


\section{References}

[1] Cosman, P.C., Rogers, J.K., Sherwood, P.G. and Zeger, K. "Combined Forward Error Control and Packetized Zerotree Wavelet Encoding for Transmission of Images over Varying Channels", IEEE Transactions on Image Processing, vol. 9, no. 6, pp 982-993, June 2000.

[2] Wu, Y., Lonardi, S. and Szpankowski, W. "Error-Resilient LZW Data Compression", IEEE Proceedings of the Data Compression Conference (DCC'06), pp. 193-202, Snowbird, UT, March 2006.

[3] Baqai, S., Baqai, F., Hameed, U., Sheikh, S. and Khokhar, A.” Error Resilience of EZW Coder for Image Transmission in Lossy Networks", Proceedings of the IEEE Fourth International Symposium on Multimedia Software Engineering (MSE’2002), pp. 328-333, Newport Beach, CA, USA ,2002.

[4] Morelos-Zaragoza, R. H. and Lin, S. "On Primitive BCH Codes with Unequal Error Protection Capabilities," IEEE Trans on Information Theory, vol. 41, no. 3, pp. 788-790, May 1995.

[5] Blahut, R. "Theory and Practice of Error Control Codes", Addison-Wesley, Reading, Mass, USA, 1993.

[6] Cosman, P., Rogers, J., Sherwood, P. and Zeger, K. "Combined Forward Error Control and Packetized Zerotree Wavelet Encoding for Transmission of Image over Varying Channels,” IEEE Trans. Image Process., vol.9, no. 6, pp. 982-993, June 2000.

[7] Said, A. and Pearlman, W. A.” A New Fast and Efficient Image Codec Based on Set Partitioning in Hierarchical Trees," IEEE Trans. Circuit Syst. Video Technol., vol. 6, pp. 243-250, June 1996.

[8] Mohammed, U.S. and Ramzy, S.M. "An Efficient Rate Allocation Scheme for Transmission of Image Streams over Binary Symmetric Channels" Journal of Engineering Sciences, vol. 35, No.1, pp.177-188, January 2007.

[9] Wang, Y. and Zhu, Q. F. "Error Control and Concealment for Video Communication: A Review," Proceeding of the IEEE, vol.86, no. 5, pp. 974-997, May 1998.

[10] Fischer, R., Mangold, P., Pelz, R.M., and Nitsche, G. "Combined Source and Channel Coding for Very Low Bitrate Mobile Visual Communication Systems," in Proc. Int. Picture Coding Symposium (PCS), Melbourne, Australia, pp. 231236, March 1996.

[11] Illgner, K. and Lappe, D. "Mobile Multimedia Communications in a Universal Telecommunications Network," in Proc. Visual Communications and Image Processing (VCIP), Taipei, Taiwan, SPIE, vol.2501, pp. 1034-1043, May 1995.

[12] Chaari, L., Fourati, M., Masmoudi, N. and Kamoun, L. "Image transmission quality analysis over adaptive BCH coding," WSEAS Transactions on Communications, vol. 7, no. 6, pp. 584-593, June 2008.

[13] Li, Q., Hu, R.Q. Qian, Y. and Wu, G. "Cooperative Communications for Wireless Networks: Techniques and Applications in LTE-Advanced Systems," IEEE Wireless Communications, Vol. 19, No. 2, pp. 22-29, April 2012.

[14] Chen, J. S. and Wang, J. X. "Cooperative Transmission in Wireless Networks using Incremental Opportunistic Relaying Strategy," IET Communications, Vol. 3, No. 12, pp. 1948-1957, 2009. 
[15] Tian, J., Zhang, Q. and Yu, F. "Non-Coherent Detection for Two-Way AF Cooperative Communications in Fast Rayleigh Fading Channels," IEEE Trans. COMM., Vol.59, No.10, pp. 2753-2762, October 2011.

[16] Khalaf, T. A. and Ramzy, S. M. "Decoding Scheme for Relay Networks with Parity -Forwarding Cooperation Protocol". IET Communications, Vol. 8, no. 8, pp. 1317-1324, 2014.

[17] Kim, Y. G. and Beaulieu, N. C." Exact BEP of Decode-and-Forward Cooperative Systems with Multiple Relays in Rayleigh Fading Channels," IEEE Trans. Veh. Technol, Vol.64, pp.823-828,2015

[18] Holland, J. H. "Adaptation in Natural and Artificial Systems: An Introductory Analysis with Applications to Biology, Control, and Artificial Intelligence," MIT Press, Cambridge, 1992.

[19] Hristakeva, M. and Shrestha, D. "Solving the 0-1 knapsack problem with genetic algorithms," Midwest instruction and computing symposium, University of Minnesota, Morris, April 2004

[20] Prieto, D. and Pérez-Aranda, R. "Study of Undetected Error Probability of BCH Codes for MTTFPA Analysis," IEEE 802.3bv Task Force, pp.1-11, January 2015

[21] Xu, H., Kun, H. and Honggang, W. "Adaptive FEC coding and cooperative relayed wireless image transmission," Digital Communications and Networks, vol 1, P. 213-221, 2015

[22] Zhang, L., Xu, R., Wang, K.," Research on image transmission mechanism through a multimode fiber based on principal component analysis" Optics and Lasers in Engineering, vol. 134, pp. 106197, 2020.

[23] Wu, S., Guan, Q., and Miao, Z. "A New Class of LT-Based UEP Rateless Codes for Satellite Image Data Transmission". AEU-International Journal of Electronics and Communications, vol. 122, pp. 153256, 2020.

[24] Lewis, A.S., Knowles, G. "Image compression using the 2-D wavelet transform", IEEE Trans. Image Processing, vol. 1, no 2, pp. $244-250$, 1992

[25] Gallager, R. "Low-Density Parity-Check Codes," IRE Transactions on information theory, vol.8, no. 1, pp. 21-28, 1962. 


\section{دراسة وتحليل أداء ترميز القناة لإرسال الصور عبر شبكات الترحيل التعاونية}

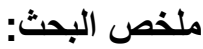

أو لاء، العمل، قمنا بفحص تأثثرات أخطاء الإرسال الناجمة عن الضوضاء على أداء نقل الصور عبر شبكات الترحيل التعاونية في وجود إمكانات مختلفة لتصحيح الأخطاء. ثانيا، يتم تحليل العلاقات بين أطوال الكود، ونسبة قوة الإشارة إلى الضوضاء (PSNR)، ونسبة الإشارة إلى الضوضاء (SNR). ثانيًا، تم تقديم المقارنة بين أداء BCH و RCPC و LDPC باستخدام SPIHT ككود مصدر . يتم تطبيق الخوارزمية الجينية، كثقنية تحسين فعالة، لحل مشكلة الطريقة المثلى لحماية الأخطاء غير المتساوية (UEP) لتقليل معدل الإرسال وتحسين جودة الصورة المرسلة. يمكن استخدام العديد من النتائج المفيدة و الهامة التي تم تقديمها في هذا العمل في تحسين أداء نقل الصور عبر الثبكات التعاونية. مقارنة بالطرق التقليدية، فإن الطريقة المقترحة تعد أكثر عملية لنقل الصور بجودة عالية عبر

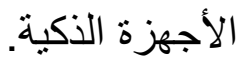

